\title{
A INSTITUCIONALIZAÇÃO DA EDUCAÇÃO PERMANENTE NO PROGRAMA DE SAÚDE DA FAMÍLIA: UMA EXPERIÊNCIA MUNICIPAL INOVADORA
}

\author{
THE INSTITUTIONALIZATION OF PERMANENT EDUCATION IN THE FAMILY HEALTH \\ PROGRAM: AN INNOVATIVE MUNICIPAL EXPERIENCE
}

I vana Macedo Cardoso 1

Ana Luiza Gualandi Murad 2

Sandra Mara Soeiro Bof 3

Resumo O Programa de Saúde da Família (PSF), adotado pela Secretaria Municipal de Saúde de Vitória (ES) como estratégia de estruturação da atenção primária, tem exigido de seus profissionais conhecimentos e habilidades em geral não valorizados em sua formação acadêmica. Além disso, a oferta constante de novos conceitos e tecnologias e a demanda da população por um serviço de saúde adequado às suas necessidades requerem do profissional da saúde aperfeiçoame nto contínuo. A Secretaria de Saúde do município, respondendo a essas necessidades, implantou em 2001 e institucionalizou em 2003 o Programa de Educação Permanente (PEP) para os profissionais de nível superior das equipes de saúde da família. Esse programa tem pautado sua atuação em uma nova concepção de educação continuada, envolvendo aprendizagem contextualizada baseada em evidências e realizada através de metodologias inovadoras, como a revisão entre pares e a aprendizagem baseada em problemas.

Palavras-chave educação permanente; institucionalização; qualidade da assistência.
Abstract The Family Health Program, which was adopted by the Municipal Health Secretariat of the city of Vitória, in the Brazilian state of Espírito Santo, as a strategy for structuring municipal primary health care, has been requiring its professionals to have knowledge and skills that are generally not stressed in their academic education. Moreover, the constant input of new concepts and technologies and the demand of the population for health services that are appropriate for their specific needs require health profe ssionals to constantly improve. The municipal health secretariat, responding to these needs, has implemented in 2001 (and institutionalized in 2003) the Permanent Education program for graduate pro fessionals of the Family Health Program staff. This program's actions have been guided by a new notion of continued education, which involves evidence-based contextualized learning that uses innovative methodologies such as peer reviewing and problem-based learning. Key words permanent education; institutionalization; quality of care. 


\section{Introdução}

Este artigo descreve e ap resenta resultados preliminares da experiência inicial de estruturação de um Programa de Educação Permanente (PEP) para o P rograma de Saúde da Família (PSF), realizado pela Secretaria Municipal de Saúde de Vitória (ES).

Capitaneado pelo Ministério da Saúde (MS) como principal estratégia para estruturação da atenção primária e adotado por muitos municípios brasileiros, o PSF fundamenta-se na promoção da qualidade de vida através de práticas de saúde centradas na família e na comunidade, gerando um novo modelo de atenção à saúde, mais justo, eqüitativo, democrático, participativo e humanizado. A execução dessas práticas exige o estabelecimento de novas relações entre os profissionais da saúde envolvidos e os indivíduos por eles atendidos, suas famílias e comunidades. Do profissional da equipe de saúde da família espera-se uma prática humanizada, competente e resolutiva, envo lvendo ações de promoção, prevenção, tratamento e reabilitação da saúde.

Estas características representam desafios à capacitação dos recursos humanos do PSF, tanto no que se re fere à formação quanto à educação continuada destes profissionais. "A formação tradicional em saúde, baseada na organização disciplinar e nas especialidades, conduz ao estudo frag mentado dos problemas de saúde das pessoas e das sociedades, levando à formação de especialistas que não conseguem mais lidar com as totalidades ou re alidades complexas" (MS, 2003). Além disso, o crescente volume de informações disponibilizado na literatura científica e a introdução de novas tecnologias exigem dos profissionais a prática da educação continuada.

O modelo dominante de educação médica continuada, constituído por cursos de atualização profissional, congressos etc., tem se mostrado ineficaz no cumprimento desta tare fa (Fox e Bennett, 1998; Cantillo e Jones, 1999; Heffner, 2001; Marins et al., 2004). Ele reproduz a abordagem das especialidades, enfoca essencialmente a atualização técnico-científica, usa uma pedagogia marcada pela transmissão vertical de conhecimentos (professor-aluno), muitas vezes fora do contex to, e não é acompanhado de uma reflexão sobre a própria prática ou de um processo de aprendizagem autodirigido e de longa duração. Geralmente, quando esses profissionais retornam aos seus serviços após cada capacitação,não conseguem aplicar o que 'ap renderam' e mu itas vezes constatam que o que ap renderam não lhes fornece elementos suficientes para enfrentar a problemática de sua realidade de trabalho. “Ta mbém são incapazes de lidar com questões complexas como a dificuldade de adesão ao tratamento, a autonomia no cuidado, a educação em saúde, o sofrimento da dor, o enfrentamento das perdas e da morte, o direito das pessoas à saúde e à informação ou a necessidade de ampliar a autonomia das pessoas" (MS, 2003). 
Diante desse contexto, e constatando lacunas na formação dos profissionais contratados para o PSF do município, a Secretaria Municipal de Saúde de Vitória buscou, em 2000, a consultoria da Escola de Saúde Pública do Ceará, instituição de renome nacional em educação para profissionais da saúde. Esta instituição, representada pelos consultores Silvia Mamede Studart Soares, Júlio César Penaforte e Henrique Luís do Carmo e Sá, desenvolveu o projeto denominado "Educação permanente para os profissionais de nível sup erior das equipes de saúde da família do município".

Tendo a Secretaria de Saúde definido a melhoria das competências clínicas dos médicos do PSF como prioridade para a educação permanente, o projeto voltava-se em sua fase inicial para esta finalidade.

A primeira etapa constituiu-se da seleção e capacitação de uma equipe de educadores médicos e do desenho de linhas de ação e estratégias do programa. A seleção dos futuros educadores foi feita pelos consultores, a partir do quadro de profissionais efetivos do município: 15 médicos especialistas nas áreas básicas de pediatria, ginecologia/obstetrícia e clínica médica, quatro enfermeiros e um odontólogo. O curso de capacitação foi dividido em cinco módulos, num total de 180 horas, iniciado em outubro de 2000 e finalizado em junho de 2001, abrangendo os conteúdos: "Fundamentos do processo de aprendizagem e educação permanente para os profissionais da saúde" e "Métodos educacionais". O eixo do modelo de educação permanente proposto era a estratégia de revisão entre pares (peer review, na literatura de origem inglesa). Outras abordagens educacionais, como aaprendizagem baseada em problemas ( $\mathrm{PBL}$, do inglês problem-based learning), eram também adotadas com finalidades específicas.

Desenhou-se, então, uma nova concepção para a educação continuada, envolvendo a aprendizagem de longa duração, a construção do conhecimento a partir das necessidades educacionais dos profissionais, com base nos problemas de sua realidade e de seu contexto, a busca de informações relevantes através da medicina baseada em evidências e a submissão dos novos conhecimentos à crítica e reflexão dos colegas.

A escolha da revisão entre pares deu-se pela observação de sua efetividade como método educacional transformador da prática dos profissionais em âmbito internacional (Beyer, 2003). Trata-se de "um processo permanente e sistemático de reflexão crítica e avaliação de sua prática profissional, conduzida por um gr upo de médicos que se organiza para tal com o intuito de obter um contínuoaprimoramento na qualidade da atenção ao paciente" (Grol e Law rence, 1995, p. 3). Dispõe de ferramentas como a construção de protocolos clínicos, análise de casos e observação da prática profissional. Esta última pode acontecer de três formas: registro em áudio, registroem vídeo e observação da atuação do médico em seu local de trabalho. 
Já a PBL constitui-se em um processo de aprendizagem organizado em torno da compreensão e da resolução de um problema. Realiza-se em pequenos grupos, coordenados por um tutor, constando de um encontro para a análise do problema, uma etapa de estudo individual e um encontro para resolução do problema. O ap rendizado é então construído a partir do co$\mathrm{n}$ h e ci mento dos participantes, orientado para problemas que se ap resentam na prática, promovendo o trabalho em equipe e também o estudo autodirigido (Schmidt, 1983, 1993; David et al., 1998; Til e Heidjen, 1998; Mamede et al., 2001).

Salientamos que, naquele momento,em 2001, a concepção de educação permanente trazida pelos consultores aproximava-se muito da definição posteriormente defendida pelo MS e hoje em fase de implantação no país, através dos pólos de educação permanente. Para os consultores, a 'educação continuada' dizia respeito ao modelo tradicional de educação feita após a gr aduação, que geralmente girava em torno de cursos pontuais, congressos etc., todos processos verticais de ensino, centrados no professor, que não levam em conta as necessidades educacionais dos profissionais e sua realidade de trabalho. Já o modelo de educação permanente proposto diferenciava-se por ser centrado na prática profissional dos educandos, propondo uma reflexão sobre esta, sua avaliação e conseqüente melhoria de performance, através das metodologias pedagógicas inovadoras descritas anteriormente. Este modelo prevê intervenções educacionais em equipe multiprofissional nas situações que demandemap erfeiçoamento de toda a equipe de saúde ou discussão dos processos de trabalho adotados.

\section{Linhas de ação}

Em julho de 2001, o PEP foi implantado para o PSF de Vitória, sendo institucionalizado em setembro de 2003 pela portaria $\mathrm{n}^{\circ}$ 021/2003 da Secretaria $\mathrm{Mu}-$ nicipal de Saúde, subordinado ao Núcleo de Recursos Humanos da secretaria. O programa teve início com 13 médicos que fizeram o curso de capacitação para educadores (pediatras, clínicos e ginecologistas) e foram distribuídos em quatro regiões de saúde que congregavam, em 2003, 52 equipes completas do PSF (médico, enfermeiro, auxiliares de enfermagem e agentes comunitários de saúde) em 15 unidades de saúde. Estas unidades possuem também assistente social, farmacêutico, psicólogo e odontólogos. Em 2004, foi criada uma coordenação de educação permanente para os enfermeiros do PSF.

Desde sua implantação, o PEP — inicialmente estruturado para os médicos - tem pautado sua atuação nas seguintes linhas de ação: Grupos de Aperfeiçoamento Profissional (GAP), Módulos de Capacitação, Treinamento em Serviço e Ambulatórios de Referência. 
Os GAP são pequenos grupos de médicos de família (sete a dez) de uma mesma região de saúde que se reúnem semanalmente em horário de trabalho, sob a supervisão de um ou dois educadores permanentes. A finalidade dos GAP é promover a reflexão, avaliação e mudança de práticas, visando à melhoria da performance clínica dos profissionais. Asmetodologias usualmente utilizadas são a revisão entre pares e o PBL. Nestasmetodologias, o educador não 'ensina', mas atua como facilitador do aprendizado, que se dá a partir dos conhecimentos prévios de cada médico.

Os temas discutidos são escolhidos pelos participantes de acordo com a realidade de sua prática clínica diária, sendo, ocasionalmente, demandados pela Secretaria. Procura-se focar cada tema em um aspecto de maior dificuldade, para que este seja analisado com maior profundidade, permitindo uma avaliação da prática de cada profissional em relação ao aspecto do tema estudado e possibilitando a fixação de metas que possam ser avaliadas posteriormente. A bibliografia utilizada nas discussões é sugerida por todos os participantes, sendo sempre baseada na melhor evidência disponível, como consensos de sociedades de especialidades médicas brasileiras, norte-americanas e européias. Dessa forma, o médico pode comparar a sua prática atual com protocolos reconhecidos, e o grupo tem a oportunidade de propor mudanças nas condutas que vinham sendo utilizadas.

Os participantes procuram contextualizar e adequar as condutas médicas à realidade local, de acordo com os recursos disponíveis na rede municipal de saúde. A resolução de um problema de saúde é sempre visto no âmbito da equipe de PSF e equipe interdisciplinar da unidade de saúde, já que uma gama enorme de agravos só é passível de resolução com o trabalho integrado da equipe de saúde, este sempre estimulado. Como, em 2004, a secretaria lançou três protocolos de atenção básica (Acolhimen to, Atenção ao pré-natal, Parto e puerpério e Saúde da criança), o espaço dos GAP foi utilizado naquele momento para treinamento destes protocolos, utilizando para isto a discussão de casos clínicos das equipes de saúde da rede e a resolução destes, comparada com as orientações dos protocolos.

Os Módulos de Capacitação em temas específicos foram introduzidos a partir de 2003, com o propósito de permitir a aquisição de conhecimentos básicos para a abordagem dos problemas mais relevantes, buscando-se, dessa maneira, facilitar a superação de lacunas mais significativas na base de conhecimentos dos médicos das equipes. Consistem em aulas dialogadas ministradas pelos educadores ou porexperts convidados, provenientes de instituições de ensino locais, para grupos maiores de médicos de família (em torno de 25). Os módulos abordam temas básicos da atenção primária (por exemplo, a consulta de puericultura) ou assuntos do domínio de especialistas, como eletrocard i ograma, além de tópicos demandados pela Secretaria de Saúde do município ou pelo MS. 
Na nossa análise, esta forma de capacitação tem se mostrado como a menos efetiva das linhas de ação adotadas, trazendo pouco impacto na prática dos profissionais. Ela recebeu a pior avaliação dos médicos em pesquisa realizada sobre as metodologias utilizadas, apesar de muitas vezes ser necessária para o repasse de informações pontuais e verticalizadas, como programas de governo. Creditamos a baixa efetividade ao método de aula expositiva, ao tamanho dos grupos (maior que o habitual dos GAP) e ao fato de as palestras nem sempre leva rem em conta a realidade do trabalho e a opinião dos educandos sobre as propostas de trabalho apresentadas.

No Treinamento em Serviço, o educador freqüenta o ambulatório do médico de família após avaliação de necessidades educacionais, realizando treinamento em sua especialidade. Esta abordagem visa, em particular, ao desenvolvi mento de habilidades clínicas, somente possíveis através de estratégias que envol vam a prática supervisionada. Exemplificando: o educador ginecologista freqüenta o ambulatório do médico de família nos dias em que este atende em saúde da mulher, orientando-o em ginecologia e obstetrícia. $\mathrm{O}$ educador pediatra atua no dia do atendimento em puericultura, e o clínico, no dia de atendimento de hipertensão arterial e diabetes. O educador utiliza técnicas de observação mútua da prática e de feedback, preconizadas na metodologia da revisão entre pares.

Esta atividade é a que demanda maior tempo na agenda do educador, por vários fatores, como o caráter individual do treinamento e a alta rotatividade dos profissionais devido ao vínculo temporário de trabalho. $\mathrm{O}$ tre inamento dura o tempo que for necessário para que o médico adquira as habilidades necessárias ao cumprimento dos programas de saúde adotados em média quatro meses para cada uma das três especialidades. Esta atividade foi a mais bem avaliada pelos profissionais médicos.

Os educadores atendem em Ambulatórios de Referência os pacientes encaminhados pelos médicos de família, quando estes sentem dificuldade em conduzir os casos. O educador pode resolver a situação clínica ap re sentada e contra-referenciar o paciente ao médico de família, retornando o paciente ao médico que o encaminhou, ou, se necessário, encaminhá-lo para um atendimento de maior complexidade. Consideramos esta atividade essencial para dar maior resolubilidade às equipes de PSF no âmbito da atenção básica. Ela também serve como espaço de manutenção das habilidades clínicas dos educadores.

Os enfermeiros também se utilizaram da estratégia dos GAP, adotando, na maioria das vezes, a metodologia PBL e trabalhando temas como a sistematização da assistência de enfermagem, o diagnóstico de enfermagem na atenção básica e o treinamento dos protocolos da secretaria. As coordenadoras do programa também realizam treinamento em serviço nas unidades de saúde nas áreas de coleta de preventivo e puericultura. 
Seguindo as diretrizes da Política Nacional de Educação Permanente em Saúde, instituída em fevereiro de 2004 pela portaria $n^{\circ} 198$ (MS, 2004), teve início,em abril do mesmo ano, o processo de educação permanente em gr upos interdisciplinares, sob a coordenação dos profissionais da equipe do Núcleo de Recursos Humanos da secretaria, composta por médicos e enfermeiros educadores, psicólogo, assistente social e pedagoga. As equipes de saúde das unidades do PSF foram divididas em grupos por região de saúde, formados por médicos, enfermeiros, assistentes sociais, farmacêuticos, psicólogos, odontólogos e coordenadores de Unidade de Saúde, em torno de 25 participantes por grupo.

Os grupos interdisciplinares têm como objetivo criar oportunidades para discussão do processo de trabalho em temas do campo de ação de toda a equipe, como interdisciplinaridade, visita domiciliária, educação em saúde e aconselhamento em DST/Aids. Cada profissional tem a oportunidade de contribuir com seu 'núcleo' de saber para a resolução de um problema que tem um 'campo' comum de atuação em toda a equipe. Na opinião da maioria dos profissionais participantes, esta atividade é importante e traz impacto na prática das equipes. Para a equipe de educadores, esta atividade educativa é importante para discutir temas que são comuns a todas as equipes e que às vezes necessitam de assessoramento de especialistas em alguns temas, como técnicos da secretaria responsáveis por programas de saúde específicos.

Porém, em nossa avaliação, esta modalidade educativa não atinge o impacto desejado na transformação das práticas próprias de cada equipe de PSF e parece haver a necessidade de uma abordagem educativa de cada equipe, em particular - e em sua totalidade —, incluindo os auxiliares de enfermagem e agentes comunitários de saúde e, em algumas situações, toda a equipe de saúde das unidades, nas quais sejam identificados os nós críticos de seu processo de trabalho e construídas soluções para estes problemas com os profissionais da saúde, gestores e comunidade.

Alguns profissionais da equipe do Núcleo de Recursos Humanos começaram a participar, no final de 2003, das reuniões do Pólo de Educação Permanente do estado do Espírito Santo e têm trazido como demanda a adequação do PEP aos moldes da proposta do MS. Estão sendo discutidas e elaboradas no núcleo estratégias educacionais que se ap roximem ainda mais das características de educação preconizadas pelo ministério: educação transdisciplinar, ascendente e descentralizada.

\section{Resultados}

O município de Vitória possui 302.633 habitantes e contava, ao final de 2003, com $44,2 \%$ de cobertura do PSF (com plano de estendê-lo a $100 \%$ da rede 
municipal de saúde até 2007), 52 equipes distribuídas em 15 Unidades de Saúde. O PEP atua em todas as equipes. Em 2003, cada médico foi submetido, em média, a 149 horas de capacitação, sendo 117 horas em módulos e GAP e 32 horas em treinamento em serviço.

Como todo projeto inovador - e que implica custos para uma instituição - , o PEP necessita ser avaliado periodicamente. Por dificuldades na consolidação de dados que gerassem indicadores de saúde mensuráve is, a coordenação do programa procurou algumas alternativas de avaliação.Uma delas foi a aplicação periódica de questionários de avaliação do programa aos médicos e enfermeiros. Através desse instrumento, aplicado no final de 2003, evidenciou-se que 94,4\% dos profissionais consideraram o PEP "um instrumento que modifica a performance clínica". Cada uma das atividades desenvolvidas pelo programa recebeu a aprovação de mais de $90 \%$ dos profissionais. $\mathrm{O}$ treinamento em serviço teve a melhor avaliação dos médicos de família: mais de $95 \%$ deles o classificaram como bom ou ótimo. As reuniões de GAP foram mais bem avaliadas que os módulos, pelas razões já analisadas.

A equipe do Núcleo de Recursos Humanos estuda a elaboração de um tipo de avaliação que considere o desempenho das equipes no cumprimento de metas pactuadas com os gestores no planejamento local de saúde e a participação do PEP neste processo.

Um outro formato de avaliação foi construído a partir da constatação do númeroexcessivo de solicitações de exame radiológico de seios da face pelos médicos da rede. Em 2002, foi elaborada uma estratégia de capacitação em rinossinusite nos GAP, através da revisão entre pares, e monitorado o número de raios $\mathrm{X}$ de seios da face solicitados pelos médicos de família e realizados na Policlínica de São Pedro, antes e depois da capacitação. Os resultados apurados demonstram uma diminuição de, em média, 40\% no número de raios $\mathrm{X}$ de seios da face solicitados nos três meses posteriores à capa citação, como se pode ver no Gráfico 1 .

Gráfico 1

Prog ressão do número de exames radiológicos de seio de face, na Policlínica de São Pedro (junho-outubro de 2002)

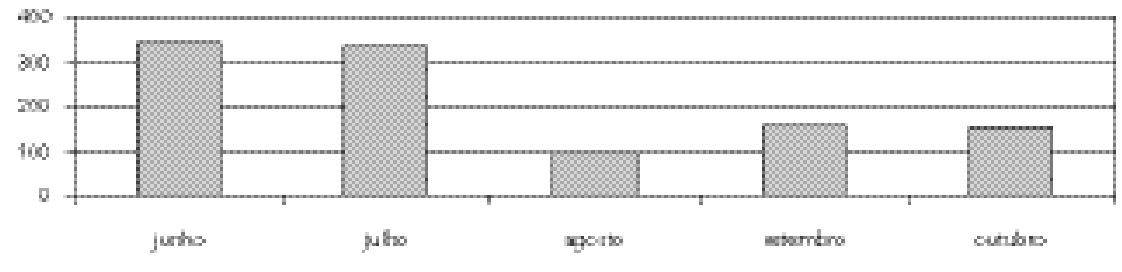

Fonte: Serviço de Radiologia da Policlínica de São Pedro 
Com a grande rotatividade de profissionais nas equipes do PSF, iniciouse em 2004 a utilização de um questionário de avaliação inicial, abordando aspectos relativos aos seus conhecimentos e habilidades clínicas nas áreas básicas de saúde da criança, saúde da mulher e saúde do adulto. Posteriormente, após treinamento, o mesmo questionário é aplicado com a finalidade de quantificar as mudanças de performance alcançadas. Aguardamos atingir um número maior de questionários respondidos para a consolidação destes dados.

Os educadores consideram o programa transformador de práticas, na medida em que capacita os profissionais da equipe de saúde a realizar os programas propostos, promovendo ganho de conhecimentos e habilidades clínicas necessárias. Constatam também que os médicos que chegam às equipes de PSF do município sem as competências necessárias para prestar um atendimento de atenção básica de qualidade à população apresentam uma melhoria nítida de performance após o treinamento em serviço, aprimorada continuamente pelas reuniões de GAP e pela atuação dos Ambulatórios de Referência.

\section{Conclusão}

O PSF requer dos profissionais competências até pouco tempo esquecidas no processo de formação profissional. A educação continuada tradicional não tem se mostrado capaz de corresponder a estas necessidades.

O processo educacional deve possibilitar aos profissionais desenvolver habilidades ap ropriadas e adaptáveis a um contexto também em constante mudança. A Secretaria Municipal de Saúde de Vitória, ciente da importância de uma educação permanente efetiva para seus profissionais, ofe rece a eles uma oportunidade ímpar de estudo e tre in a mento dentro do horário de trabalho, através do PEP. Este tem se mostrado eficiente na capacitação clínica dos médicos e enfermeiros do PSF, melhorando a qualidade do atendimento à população.

A incorporação da estratégia de educação permanente, lançada pelo MS em 2004, é o novo desafio do programa, que busca a construção de novas metodologias educacionais ainda mais abrangentes. O PEP deve avançar, visando a promover a integração dos profissionais das equipes de saúde, assim como a avaliação e a transformação de seus processos de trabalho. 


\section{Notas}

1 Educadora do Programa de Educação Permanente do Programa de Saúde da Família (PEP-PSF) da Prefeitura Municipal de Vitória (ES). Médica Pediatra, Especialista em Família pelas Faculdades de Vitória (FDV). <ivanacardoso@terra.com.br>

2 Educadora do Programa de Educação Permanente do Programa de Saúde da Família PEP-PSF da Prefeitura Municipal de Vitória. Médica Ginecologista, Especialista em Oncologia Ginecológica pela Universidade Federal do Espírito Santo. <ana_luiza_g@hotmail.com>

3 Educadora do Programa de Educação Permanente do Programa de Saúde da Família PEP-PSF da Prefeitura Municipal de Vitória. Médica Ginecologista. <sabof@terra.com.br>

\section{Re ferências}

BEYER, Martin et al. 2003. The development of quality circles/peer re vi ew groups as a method of quality improvement in Europe: results of a survey in 26 European countries. Family Practice, v. 20, n. 4 , p. 443-451.

CANTILLO, Peter; JONES, Roger. 1999. Does continuing medical education in general practice make a difference? British Medical Journal, n. 318, p. 1276-1279.

DAVID, Tim J. et al. 1998. Problem-based learning as an alternative to lecture - b ased continuing medical education. Jour nal of the Royal Society of Medicine, v. 91, p. 526-630.

FOX, Robert. D.; BENNETT, Nancy L. 1998. Continuing medical education: learning and change: implications for continuing medical education. $B$ ritish Medical Journal, n. 316, p. 466-468.
GROL, Richard; LAWRENCE, Martin, 1995. Quality improvement by peer review. Oxford: Oxford University Press.

HEFFNER, John. E. 2001. Altering physician behavior to improve clinical performance. Topics in Health Information Management, v. 22, p. 1-9.

MAMEDE, Silvia et al. 2001. Aprendizagem baseada em problemas. Fortaleza: Hucitec.

MARINS, João José N. et al. 2004. Educação médica em transformação. São Paulo: Abem/Hucitec.

MAZMANIANN, Paul E.; DAVIS, David A. 2002. Continuing medical education and the physician as a learner: guide to the evidence. Journal of American Medicine Academy, v. 288, p. 1057-1060.

MS (Ministério da Saúde). 2003. Caminhos para a mudança da formação e desenvolvimento dos profissionais de saúde: 
diretrizes para a ação política para assegurar Educação Permanente no SUS. Disponível em: <portal.saude.gov.br/ saude/arquivos/pdf/Caminhos_para_a_ mudança2.pdf $>$. Acesso em: 20 mar. de 2003.

2004. Portaria no. 198 , de 13 de fevereiro de 2004. Institui a Política Nacional de Educação Permanente em Saúde como estratégia do Sistema Único de Saúde para formação e o desenvolvimento de trabalhadores para o setor e dá outras providências.

SMS-Vitória (Secretaria Municipal de Saúde de Vitória). 2003a. Portaria no. 021/ 2003, de 16 de setembro de 2003. Institui o Programa de EducaçãoPermanente para médicos e enfer meiros do Progr ama de Saúde da família de Vitória 2003b. Relatório final de atividades do Programa de Educação Permanente. Vitória: Secretaria Municipal de Saúde.
SCHMIDT, Henk. G. 1983. Problem-based learning: rationale and description. $\mathrm{Me}$ dical Education, n. 17, p. 11-16.

1993. Foundations of problem-based learning: some explanatory notes. Medical Education, v. 27, p. 422-432.

SMITH, C. Scott; IRBY, David M. 1997. The roles of experience and reflection in ambulory care education. Academic Medicine, v. 72, n. 1, p. 32-35.

SOUSA, Maria de Fátima; NETO, Milton M. da C. 2000. Educação Permanente. Cadernos da Atenção Básica, n. 3, p. 9-20.

TIL, Cita van; HEIJDEN, Francy van der. 1998. PBL study skills. Maastricht: Vakgroep O\&O.

Recebido em 15/10/2004

Aprovado em 13/06/2005 